\title{
Unusual case of complex fracture dislocation of the elbow
}

\author{
Rebeca Díaz Suárez ${ }^{1}$ (1) . Gonzalo Luengo Alonso ${ }^{1}$. Miguel Ángel Porras Moreno ${ }^{1,2}$. Verónica Jiménez Díaz ${ }^{1,2,3}$. \\ Lorena García Lamas ${ }^{1,2} \cdot$ Navidad Cecilia López ${ }^{1,2,3}$
}

Received: 30 July 2018 / Accepted: 1 November 2018

(C) The Author(s) 2018

\section{Introduction}

In terms of major joints, the elbow is the second most frequently dislocated joint in adults (7.3\%). Only shoulder dislocation is more common (50.6\%) [1]. It represents up to $3-6 \%$ of elbow injuries in children and is the most commonly dislocated joint among this group [2]. Up to 10-20\% of injuries affecting the elbow are dislocations: They are classified as simple or complex, based on the presence of a fracture [3].

This injury frequently occurs during sporting activities, patient falling onto an extended hand with the elbow in extension and abduction. However, elbow fracture dislocation may occur in distinct patterns.

Complex dislocations are related to a high number of complications, including recurrent instability, arthrosis, stiffness, infection, nerve and vascular injury, compartment syndrome and heterotopic ossification [3].

The most frequent complication related to this injury is extension loss; early mobilization can prevent this from happening, obtaining a good and functional range of motion (ROM). It is important to bear in mind: Most daily activities can be performed with a 30-130 degrees ROM [4].

Up to $20 \%$ of elbow dislocations are complex, requiring surgical treatment to achieve proper stability and a good outcome $[4,5]$ First of all, closed reduction should be performed in the emergency department, followed by open reduction and internal fixation [6]. The main objective treating elbow fracture dislocations is to achieve stability so

Rebeca Díaz Suárez

rebecadiazsuarez@gmail.com

1 Hospital Universitario 12 de Octubre, Servicio Cirugia Ortopedica Y Traumatologia, Planta 7 Residencia General Av Cordoba SN Km 5400, Madrid, Spain

2 Hospital Universitario 12 de Octubre, Unidad Mano y Codo, Madrid, Spain

3 Departamento de Cirugía, Universidad Complutense, Madrid, Spain patients start therapy as soon as possible. With early therapy, we are trying to avoid stiffness.

We are reporting a very rare case of complex elbow dislocation, involving ulnar diaphysis, coronoid process and radial head fractures.

\section{Case report}

A 41-year-old man, with no previous medical records, came into our emergency department after being involved in a motor vehicle accident complaining of pain and swelling in his left elbow and right hand.

He had marked swelling over his left elbow and forearm, and Nelaton's triangle was not defined. Neurovascular status was physiological, and there were no associated wounds. In his right hand, third and fourth proximal inter-phalanx joints were dislocated.

Plain radiographs of the elbow, forearm and right hand were obtained (Fig. 1), showing posterolateral complex fracture dislocation of the elbow. His right hand showed dorsal proximal inter-phalanx dislocation, affecting his third and fourth fingers. Closed reduction was performed without any complications, and radiological images were obtained to check articular congruence in both elbow and hand (Fig. 2). After closed reduction, CT scan showed a very rare fracture pattern: ulnar diaphyseal fracture, coronoid process and displaced radial head fracture (Fig. 3). This pattern does not correspond to any previously described elbow dislocation, bifocal ulnar fracture and radial head fracture.

The patient was admitted to our center, and he had surgery the following day. Open reduction and internal fixation of the ulna was initially performed. Locking compression plate was implanted, through a direct posterior approach to the ulna. Once the ulnar fracture was fixed, lateral approach to the radial head was done to treat radial head fracture. Comminution affecting radial head fracture was observed, approximately $25 \%$, and resection was performed with no prostheses implanted. 

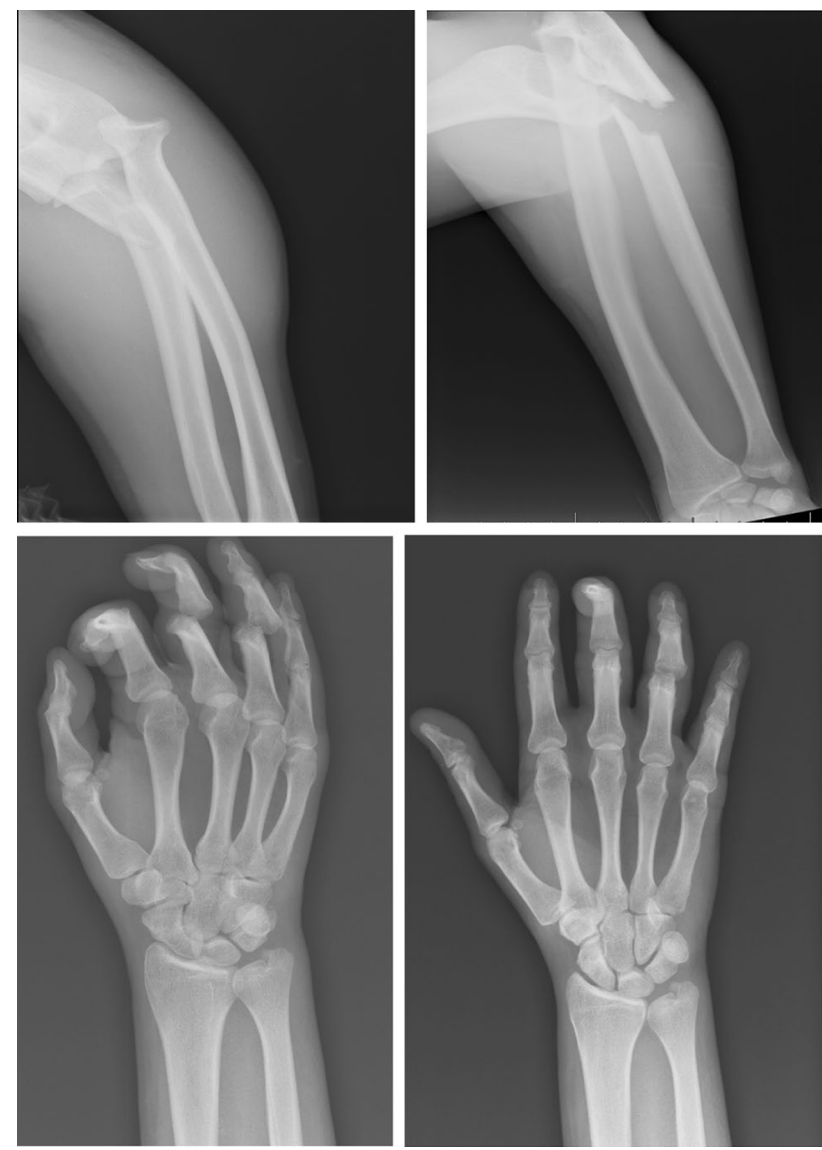

Fig. 1 Initial fractures

After radial head resection, coronoid process fracture was evaluated. No osteosynthesis was possible, as it was a small piece of bone, so we only reattached the anterior capsule. The annular ligament was also repaired, and finally, the ulnar collateral ligament was repaired and reattached.

The elbow was easily dislocated, even with 110 degrees flexion. After checking joint instability, we decided to implant an external fixation, using two pins to distal humerus and another two to the ulnar, further away from the fracture site (Fig. 4). The external fixation was fixed at 90 degrees checking articular congruence with X-rays.

He presented postoperative ulnar neuropathy that was recovered progressively in 1 month. He was discharged 2 days after surgery. External fixator removal was 2 weeks later. Therapy to his fingers started the day after surgery and to his elbow right after the external fixation was removed. Bone consolidation was assessed by physical examination, X-rays and no pain at fracture sites. It took 6 weeks to achieve consolidation (Fig. 5).

Currently, after 2 years, our patient is pain free, with 10-140 degree ROM. His analog visual scale is 0 , and his DASH score is 25.83 . He is back to his previous daily activities and sports with no associated limitations (Fig. 6).

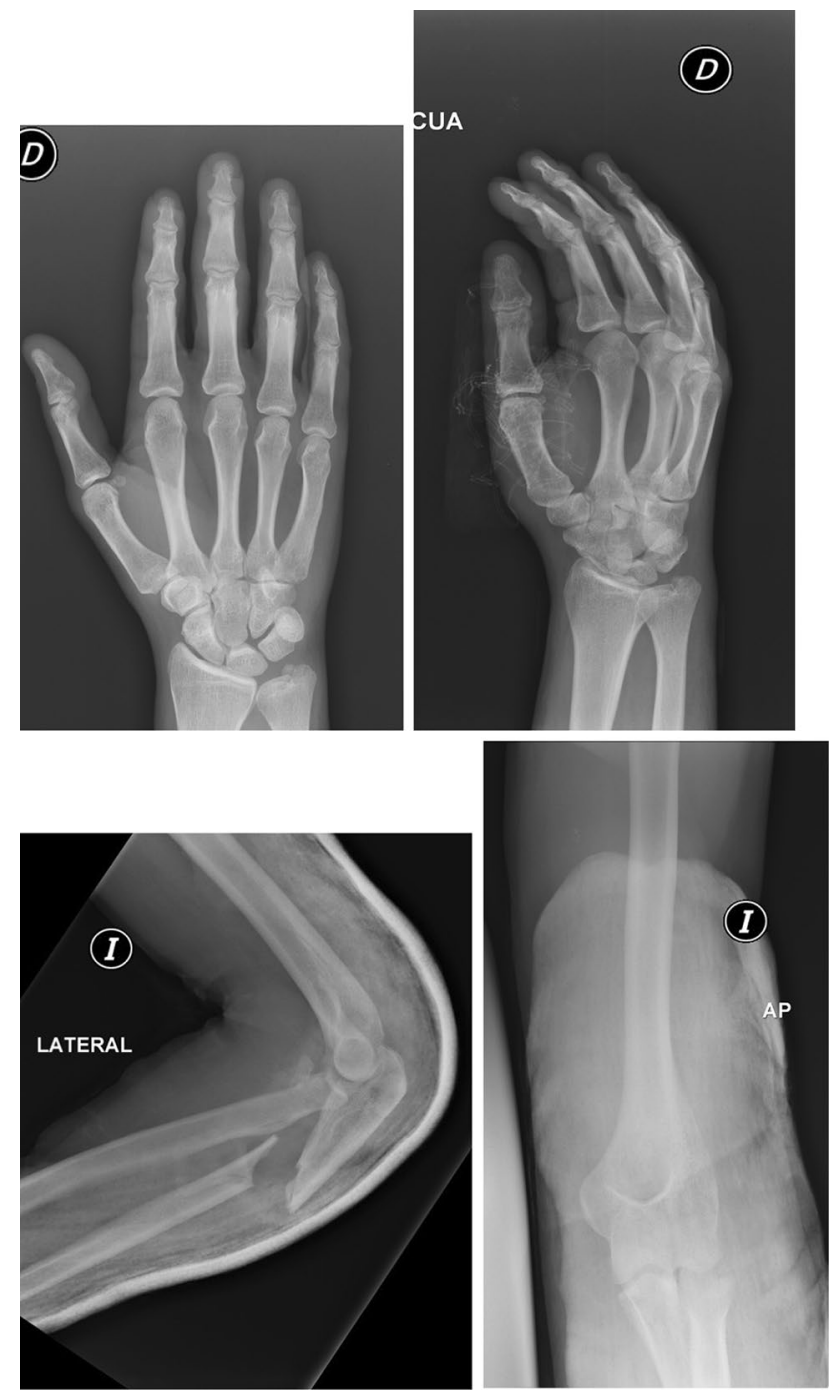

Fig. 2 Fractures after closed reduction

\section{Discussion}

Elbow joint stability relies on primary stabilizers (ulnohumeral articulation, lateral collateral ligament complex and medial collateral ligament complex) and secondary stabilizers (radiohumeral articulation, capsule and muscles that cross the joint). All these structures work together providing stability to the joint $[3,4,7]$.

Elbow instability can be simple (no associated fracture), or complex, in which fractures are concurrently associated with dislocation [7, 8]. More than $20 \%$ of elbow dislocations are related to fractures [9]. Three main patterns of complex elbow instability have been described: posterolateral fracture dislocation, also known as the terrible triad, varus posteromedial in which anteromedial coronoid fracture with ligament complex disruption occurs, 

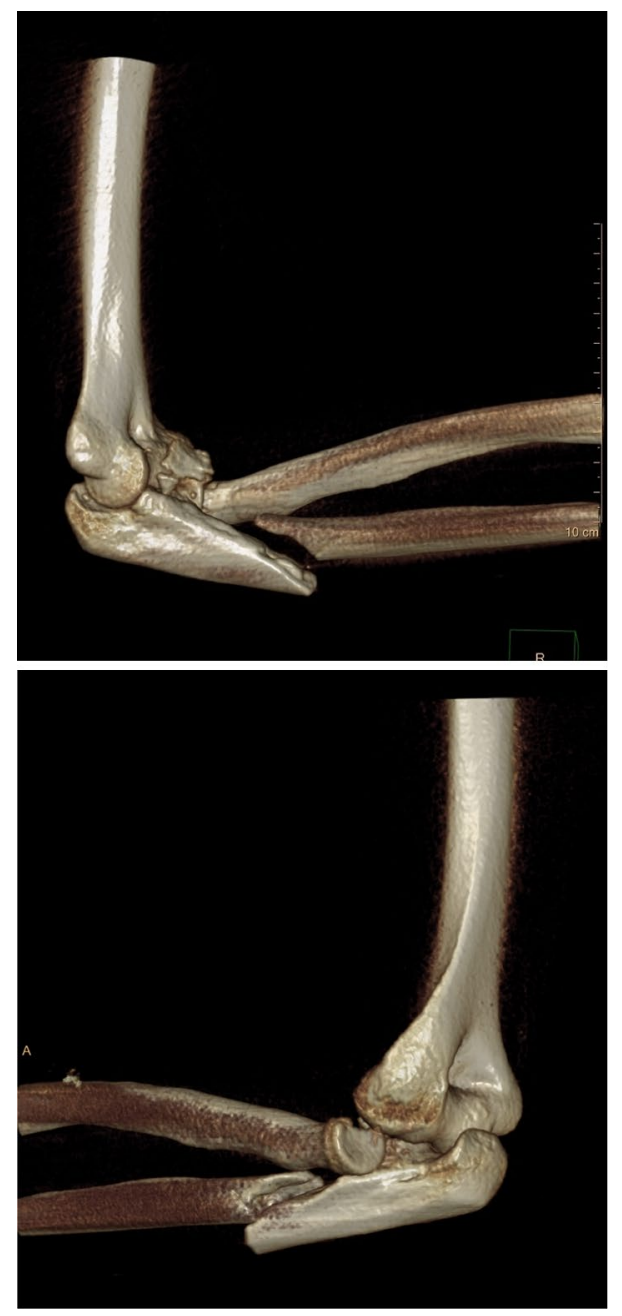

Fig. 3 CT scan

and trans-olecranon fracture dislocations [6]. Another type, ulnar diaphyseal fracture associated with radial head dislocation, is known as Monteggia fracture dislocations [10-13].

Bado classified Monteggia lesions into four groups depending on the direction of the radial head dislocation and the angulation of the ulna fracture [14]. Later on, Jupiter classified Bado type II into groups: IIA with coronoid process and distal olecranon fractures, IIB with ulna fracture in the transition from metaphysis to diaphysis, IIC with ulna diaphysis fracture and IID with olecranon fracture which extends to the proximal half of the ulna $[8,9]$.

Bado described Monteggia-like lesions, which differ in radiological appearance, but are similar in injury mechanism.

High-energy mechanisms could create a different fracture pattern, combining the patterns above indicated. Fracture pattern combination is a rare situation. These combinations require special considerations, as different instability patterns could simultaneously be present $[11$, $12,15]$.

The present case shows a fracture dislocation pattern unrelated to any previously described elbow dislocation, bifocal ulnar fracture and radial head fracture. It could also be classified as a combination of the terrible triad and a type IIC according to Jupiter's classification of type II Monteggia fracture dislocation. Different fracture patterns are associated with different ligament damages.

As it is a very rare fracture dislocation pattern, there is no standardized protocol available in terms of surgical treatment. However, it is well known that main objective is to provide sufficient stability to begin elbow therapy. Early elbow motion avoids complications as stiffness and heterotopic ossification, providing patients a functional joint. But, trying to achieve early motion could create instability, so an adequate treatment should be performed to achieve success.

A review of the literature shows there are many different strategies for these injuries $[11,12,16]$. Due to the lack of information, this case represents a surgical challenge.

In our case, we always start treating bone fractures. Initially, we fixed the ulnar diaphysis fracture with $3.5-\mathrm{mm}$ limited contact dynamic compression plate, applied to the posterior surface of the ulna through a posterior approach as Laun et al. [11] recommended. We recommended using the less fractured fragment so we could re-establish ulnar length. The most important aspects of ulnar anatomy should be sigmoid notch length and dorsal proximal ulnar curvature.

We do not attempt to identify ulnar nerve, as this structure does not interfere unless an anteromedial fragment from coronoid process is present. If anteromedial fragment is present, we identify the ulnar nerve without transposition.

The main issue with fracture fixation was whether to treat radial head fracture or not. Egol et al. [13] showed that resection and radial head fixation have similar elbow function. Nevertheless, Laun et al. [11] recommended reconstruction or prosthetic replacement. We think that it has to be evaluated during surgery as radial head is a secondary stabilizer and plays an important role in the absence of the medial collateral ligament complex. Finally, we decided to excise radial head fragment as we thought that instability was not related to it. Fixation was not possible due to the small bone size.

As we decided to excise radial head, better surgical view of coronoid process was obtained; this is why coronoid process was the last fracture treated. Elbow instability could be related to coronoid fractures. In cases with large fragments, the literature recommended open reduction and internal fixation [11-13, 16, 17]. If small coronoid process fragment is present, bone fixation would be not possible [16, 17]. In these cases, anterior capsule should be repaired to prevent elbow dislocation. As our fracture had a very small 
Fig. 4 Postoperative images
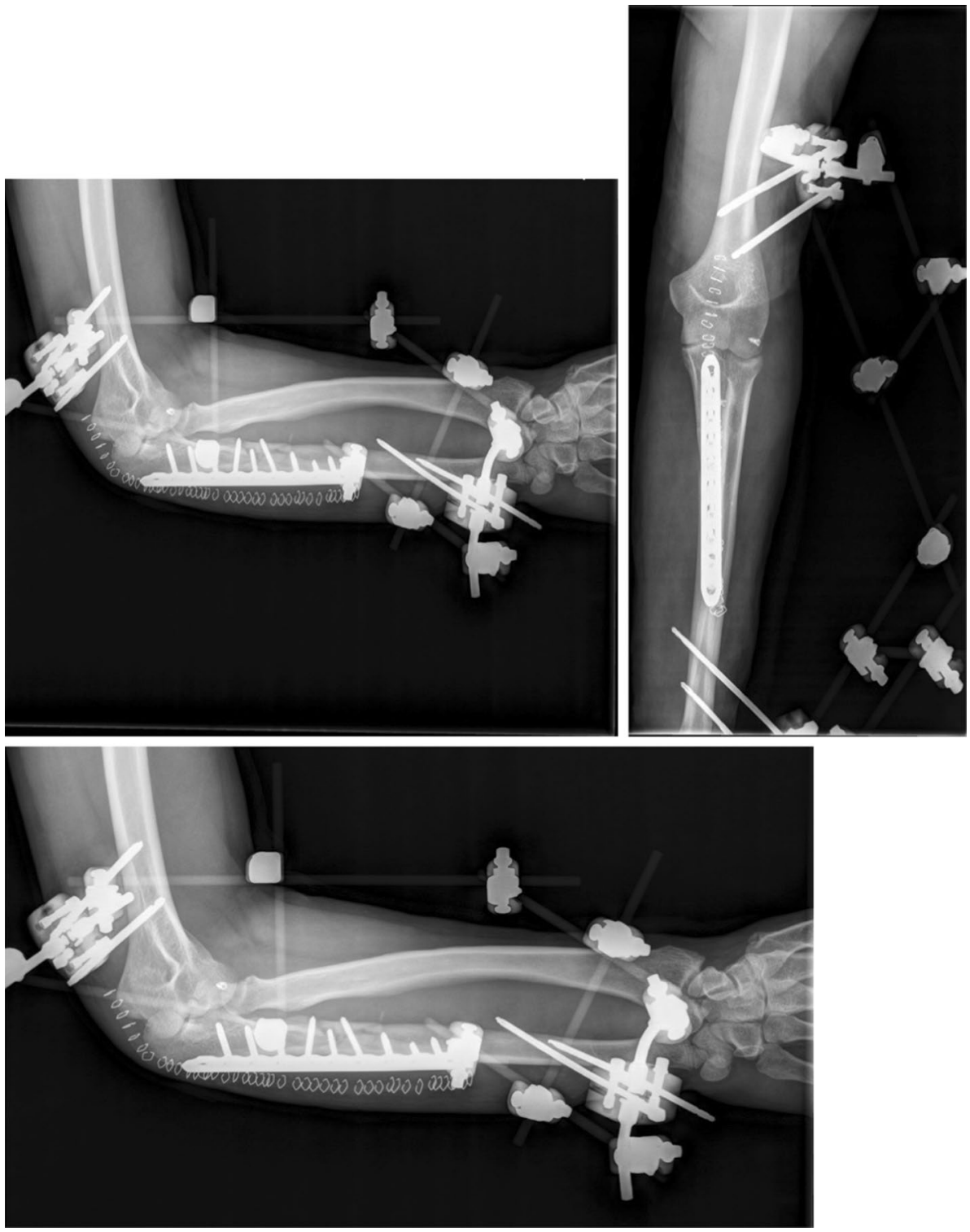
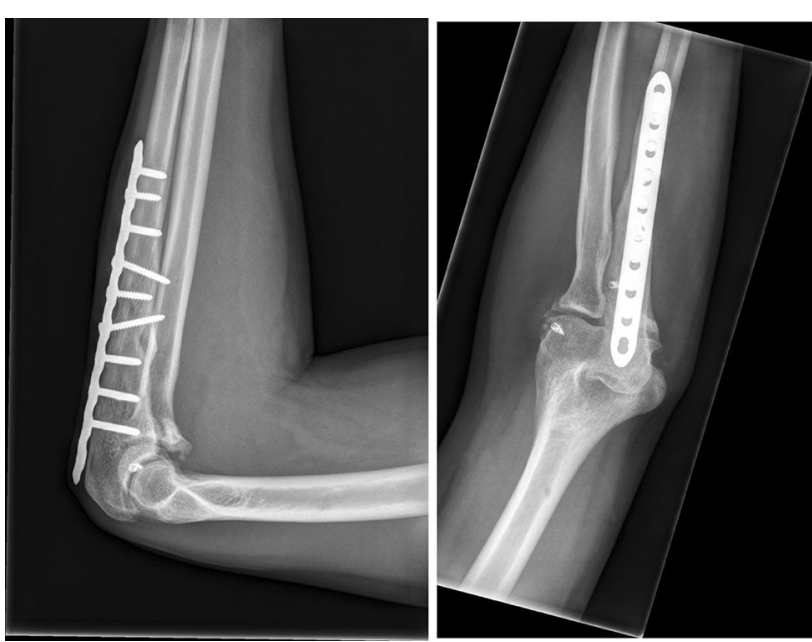

Fig. 5 Fracture healed coronoid process, we decided not to fix it and repair the anterior capsule.

After the bone fixation, the collateral ligament was repaired using an anchor to the lateral epicondyle. After all repairs were done, the elbow was still unstable even with elbow flexion, so placing an external fixator was mandatory.

Neal et al. [17] recommended smaller caliber pins to reduce iatrogenic damage to articular surface. We used two pins in the distal humerus and another two further away from fracture site, avoiding pins through the articular surface.

Stiffness is highly related to immobilization, so external fixation should be long enough to achieve stabilization and, at the same time, short enough to avoid stiffness. Some authors recommended that the elbow fixator should be kept locked for only 7 days, after that start continuous passive movement and remove elbow fixator after 6 weeks [12]. However, there are different opinions about how long the external fixator 


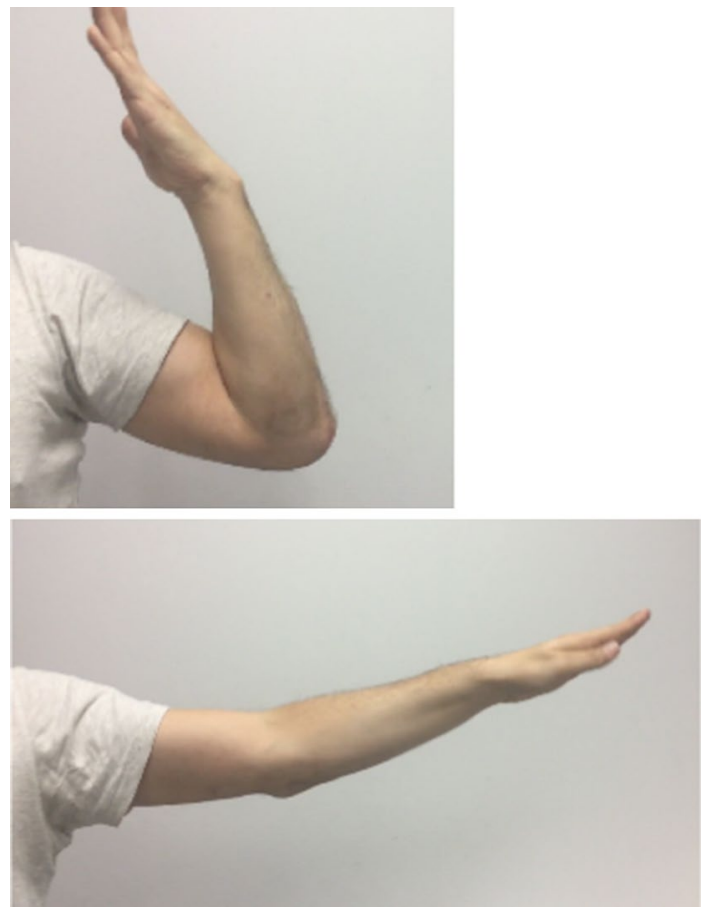

Fig. 6 Elbow range of motion

should remain in. Tan et al. maintained external fixator for two months, Knoch et al. for 7.6 weeks and Zeiders et al. for 6 weeks [14, 17-22].

We maintained external fixation locked for 2 weeks, and then under anesthesia, we checked elbow stability before pin removal.

Therapy started, with passive ROM, the day after pin removal. Initial therapy was performed with the forearm in pronation and elbow flexion. Non-weight lifting or contact sports were authorized until 12 weeks post-surgery.

In conclusion, each different injury pattern is associated with multiple joint instabilities, which require different managements. Associated instabilities require adequate fracture fixation and soft tissue repair to achieve a stabilized and functional joint.

Acknowledgements We are thankful to the patient depicted in this report (identity classified due to ethical issues). We are also indebted to Talithia Richards for reviewing the English manuscript.

\section{Compliance with ethical standards}

Conflict of interest We have no conflicts of interest to disclose.

Ethical approval All procedures performed in studies involving human participants were in accordance with the ethical standards of the institutional and/or national research committee and with the 1964 Helsinki declaration and its later amendments or comparable ethical standards.
Informed consent Informed consent was obtained from the patient included in the case report.

Open Access This article is distributed under the terms of the Creative Commons Attribution 4.0 International License (http://creativeco mmons.org/licenses/by/4.0/), which permits unrestricted use, distribution, and reproduction in any medium, provided you give appropriate credit to the original author(s) and the source, provide a link to the Creative Commons license, and indicate if changes were made.

\section{References}

1. Hindle P, Davidson E, Biant L, Court-Brown C (2013) Appendicular joint dislocations. Injury 44(8):1022-1027

2. Kushwaha SS, Kumar M, Shantanu K, Kumar D (2017) Isolated pure lateral dislocation of elbow in a 9-year-old child: a rare case. Rep J Orthop Case Rep 7(5):89-91

3. Gottlieb M, Schiebout J (2018) Elbow dislocations in the emergency department: a review of reduction techniques. J Emerg Med 54(6):849-854. https://doi.org/10.1016/j.jemermed.2018.02.011

4. Jones A, Jordan R (2017) Complex elbow dislocations and the "Terrible Triad" injury. Open Orthop J 11(Suppl-8, M7):1394-1404. https://doi.org/10.2174/1874325001711011394

5. Ando A, Hagiwara Y, Koide M, Yamashiro M, Matsuda M, Itoi E (2017) Lateral dislocation of the elbow with concomitant lateral epicondyle fracture: a case report and review of the literature. J Orthop Sci. https://doi.org/10.1016/j.jos.2016.12.028

6. Morrey BF (2009) Current concepts in the management of complex elbow trauma. Surgeon 7:151-161

7. Aquilina A, Grazette A (2017) Clinical anatomy and assessment of the elbow. Open Orthop J 11(Suppl-8, M2):1347-1352

8. Waymack JR, An J (2018) Dislocation, elbow, posterior. StatPearls [Internet]. StatPearls Publishing, Treasure Island

9. De Haan J, Schep N, Tuinebreijer W, den Hartog D (2010) Complex and unstable simple elbow dislocations: a review and quantitative analysis of individual patient data. Open Orthop J 4:80-86 PMID:20361035

10. Suarez R, Barquet A, Fresco R (2016) Epidemiology and treatment of Monteggia lesion in adults: series of 44 cases. Acta Ortop Bras 24(1):48-51

11. Laun R, Wild M, Brosius L, Hakimi M (2015) Monteggia-like lesions - treatment strategies and one-year results. GMS Interdiscip Plast Reconstr Surg DGPW. https://doi.org/10.3205/iprs000072

12. Jungbluth P, Tanner S, Schneppendahl J, Grassmann JP, Wild M, Hakimi M, Windolf J, Laun R (2018) The challenge of Monteggialike lesions of the elbow: mid-term results of 46 cases. Bone Joint $\mathrm{J}$ 100-B(2):212-218. https://doi.org/10.1302/0301-620X.100B2.BJJ2017-0398.R2

13. Egol KA, Tejwani NC, Bazzi J, Susarla A, Koval KJ (2005) Does a Monteggia variant lesion result in a poor functional outcome? A retrospective study. Clin Orthop Relat Res 438:233-238. https://doi. org/10.1097/01.blo.0000168806.79845.8b

14. Wyrick JD, Dailey SK, Gunzenhaeuser JM, Casstevens EC (2015) Management of complex elbow dislocations: a mechanistic approach. J Am Acad Orthop Surg 23(5):297-306

15. Rijal L, Kc KM, Sagar G (2012) Elbow dislocation with ipsilateral radius and unla fracture: Is it so common? Nepal Med Coll J 14(2):163-164

16. Chan K, King G, Faber K (2016) Treatment of complex elbow fracture-dislocations. Curr Rev Musculoskelet Med 9(2):185-189

17. Chen NealC (2018) Elbow fractures with instability. Hand Clin 34(1):75-83 
18. Zeiders GJ, Patel MK (2008) Management of unstable elbows following complex fracture-dislocations - the "terrible triad" injury. J Bone Joint Surg Am 90:75-84

19. McKee MD, Bowden SH, King GJ, Patterson SD, Jupitar JP et al (1998) Management of recurrent complex instability of the elbow with a hinged external fixator. J Bone Joint Surg Br 80:1031-1036

20. Von Knoch F, Marsh JL, Steyers C, McKinley T, O'Rourke M, Bottlang M (2001) A new articulated elbow external fixation technique for difficult elbow trauma. Iowa Orthop J 21:13-19
21. Sanchez-Sotelo J, Morrey M (2016) Complex elbow instability: surgical management of elbow fracture dislocations. EFORT Open Rev 1(5):183-190

22. Tan V, Daluiski A, Capo J, Hotchkiss R (2005) Hinged elbow external fixators: indications and uses. J Am Acad Orthop Surg 13:503-514 